\title{
Pre-Stroke Use of Beta-Blockers Does Not Lower Post-Stroke Infection Rate: An Exploratory Analysis of the Preventive Antibiotics in Stroke Study
}

\author{
Willeke F. Westendorp ${ }^{a} \quad$ Jan-Dirk Vermeij ${ }^{a} \quad$ Matthijs C. Brouwer ${ }^{a, b}$ \\ Y.B.W.E.M. Roos ${ }^{a}$ Paul J. Nederkoorn ${ }^{a}$ Diederik van de Beek ${ }^{a}$ b for the PASS \\ Investigators \\ ${ }^{a}$ Department of Neurology, Academic Medical Center, and ${ }^{b}$ Centre of Infection and Immunity Amsterdam, Academic \\ Medical Center, University of Amsterdam, Amsterdam, The Netherlands
}

\section{Key Words}

Cerebrovascular disease/stroke - Infection - Pneumonia .

Immune suppression · Beta-blocker

\begin{abstract}
Background: Stroke-associated infections occur frequently and are associated with unfavorable outcome. Previous cohort studies suggest a protective effect of beta-blockers (BBs) against infections. A sympathetic drive may increase immune suppression and infections. Aim: This study is aimed at investigating the association between BB treatment at baseline and post-stroke infection in the Preventive Antibiotics in Stroke Study (PASS), a prospective clinical trial. Methods: We performed an exploratory analysis in PASS, 2,538 patients with acute phase of stroke ( $24 \mathrm{~h}$ after onset) were randomized to ceftriaxone (intravenous, $2 \mathrm{~g}$ per day for 4 days) in addition to stroke unit care, or standard stroke unit care without preventive antibiotic treatment. All clinical data, including use of BBs, was prospectively collected. Infection was diagnosed by the treating physician, and independently by an expert panel blinded for all other data. Multivariable analysis was performed to investigate the relation between BB treatment and infection rate. Results: Infection, as defined by the physician, occurred in 348 of 2,538 patients
\end{abstract}

(14\%). Multivariable analysis showed that the use of BBs at baseline was associated with the development of infection during clinical course (adjusted OR (aOR) 1.61, 95\% Cl 1.192.18; $p<0.01$ ). BB use at baseline was also associated with the development of pneumonia (aOR 1.56, 95\% Cl 1.05-2.30; $p=0.03$ ). Baseline BB use was not associated with mortality (aOR 1.14, 95\% Cl 0.84-1.53; $\mathrm{p}=0.41$ ) or unfavorable outcome at 3 months (aOR 1.10, 95\% Cl 0.89-1.35; $\mathrm{p}=0.39$ ). Conclusions: Patients treated with BBs prior to stroke have a higher rate of infection and pneumonia.

(c) 2016 The Author(s) Published by S. Karger AG, Basel

\section{Introduction}

Infections frequently complicate the acute phase of stroke and have been associated with unfavorable outcome in stroke patients [1]. The high risk for post-stroke infection is at least partly driven by a stroke-induced immune suppression, which is hypothesized to be caused by increased sympathetic activity [2]. In an experimental study, administration of beta-blockers (BBs) after the onset of stroke was found to decrease the risk of infection [3]. It has been suggested that in stroke patients, administration of BBs in the acute phase after stroke could influ-

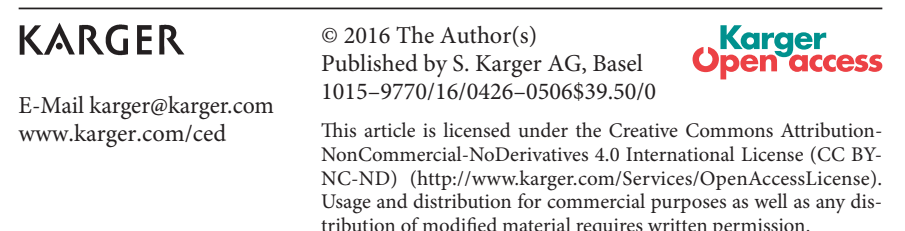

Prof. Dr. Diederik van de Beek, MD, PhD

Department of Neurology

Academic Medical Center

PO Box 22660, NL-1100 DD Amsterdam (The Netherlands)

E-Mail d.vandebeek@amc.uva.nl 
ence the immune suppression associated with acute stroke and decrease the risk of infections after stroke. Two recent cohort studies reported conflicting results on the association between BBs use and occurrence of infections in patients with acute stroke $[4,5]$.

This study is aimed at analyzing whether BB treatment influenced post-stroke infection in patients included in the Preventive Antibiotics in Stroke Study (PASS), a randomized open-label masked endpoint clinical trial on the efficacy and safety of preventive ceftriaxone in adults with acute stroke [6].

\section{Methods}

We investigated whether infection risk differs between patients treated with $\mathrm{BB}$ prior to stroke and $\mathrm{BB}$ naive patients. Therefore, all patients included in the intention-to-treat population of PASS were included in the current study. In PASS, adult patients in the acute phase of ischemic or hemorrhagic stroke (within $24 \mathrm{~h}$ after onset) with a National Institutes of Health Stroke Scale (NIHSS) score of 1 or higher, were randomized to receive ceftriaxone (intravenous, 2 g per day for 4 days) in addition to stroke unit care, or standard stroke unit care without preventive antibiotic treatment. We excluded patients with an infection at admission, using antibiotics within $24 \mathrm{~h}$ of randomization, with a known allergy to antibiotics, and patients in whom death was imminent. The trial protocol, statistical analysis plan, and main article of the study results have been published before [6]. Since the current analysis was not pre-planned in the PASS statistical analysis plan, it should be regarded as an exploratory analysis.

Baseline characteristics, clinical parameters, and endpoints were prospectively collected in case record forms that were filled out by the treating physician. Pneumonia, urinary tract infection (UTI), and other infections in the PASS were diagnosed by the treating physician and scored by an expert panel of 2 independent experts who were blinded for treatment allocation and adhered to the Centers for Disease Control and Prevention criteria [7]. Prestroke use of BBs was prospectively recorded at baseline for all patients based on the observational studies and hypotheses mentioned in the introduction. In the Netherlands, it is standard care to continue antihypertensive medication used at home during hospital admission for acute stroke.

Differences in baseline characteristics of patients with or without $\mathrm{BB}$ therapy prior to stroke are shown as percentages or mean (with SD) or median values (with interquartile range). We tested whether baseline characteristics were associated with infection overall and pneumonia and UTI separately by t test, Mann-Whitney $\mathrm{U}$ or chi-square test when appropriate. Included baseline characteristics were: age, sex, ethnicity, medical history prior to stroke (atrial fibrillation/flutter, stroke, myocardial infarction, cardiac valve disease, peripheral vascular disease, hypertension, hypercholesterolemia, pulmonary obstructive disease, diabetes mellitus, alcoholism, malignancy), medication used prior to stroke (anticoagulants, antiplatelet therapy, statins, ACE-inhibitors, BBs, proton pump inhibitors), smoking status, disability prior to stroke, phys-

Pre-Stroke Use of BBs Does Not Lower

Post-Stroke Infection Rate ical examination at admission (heart rate, systolic/diastolic RR, temperature), stroke severity (NIHSS), dysphagia, use of urinary catheter, stroke type, acute treatment (intravenous or intra-arterial thrombolysis, coagulant therapy), and randomization. Characteristics with an association in univariate analysis $(\mathrm{p}<0.05)$ were included in multivariate analysis. Variables known to have a strong association with infection were a priori included in multivariate analysis; these were: age, stroke severity, presence of dysphagia, and urinary catheterization. Association of BBs therapy and mortality at discharge and 3 months, and unfavorable functional outcome on the modified Rankin Scale (mRS 3-6) was estimated in univariate analysis and subsequent regression analysis including strong prognostic baseline variables, as described in the PASS protocol (age, stroke severity, history of stroke or diabetes, prior disability on mRS at admission). Because immune suppression is most pronounced and infection rate higher in the first days after stroke, we performed a subgroup analysis on infections within the first week. All analyses were performed with IBM SPSS statistics version 22 .

\section{Results}

From July 6, 2010 to March 23, 2014, 2,538 patients were included in PASS: $84 \%$ of patients had ischemic stroke, $11 \%$ hemorrhagic stroke, $4 \%$ transient ischemic attack, and $2 \%$ had another diagnosis. At baseline, 885 of 2,538 patients (35\%) used BBs. Baseline characteristics of these patients are shown in table 1. Patients using BBs prior to stroke were older; more often had a history of atrial fibrillation, stroke, hypercholesterolemia, hypertension, myocardial infarction, cardiac valve disease, and peripheral vascular disease and used more medication prior to stroke. Disability prior to stroke and stroke severity were similar between patients who used BBs and those who did not.

Infection, as defined by the physician, occurred in 348 patients, $130(10 \%)$ in the ceftriaxone group and 218 (17\%) in the control group. In one of these patients, baseline use of BBs was unknown; this patient was excluded from analysis. Infection was diagnosed within the first week in 270 patients by the physician and in 98 patients according to expert panel [6].

Infection rates were higher in patients using BBs at baseline, as compared to patients not using BBs (table 2). Adjusted OR (aOR) for the use of BBs at baseline and post-stroke infection was 1.61 (95\% CI 1.19-2.18; p < 0.01 ; table 3); for the expert panel definition for infection aOR was 1.64 (95\% CI 1.08-2.50; $\mathrm{p}=0.02)$. Additional analyses restricted to infection occurring in the first week after stroke showed similar results (data not shown).

$\mathrm{BB}$ use at baseline was associated with stroke-associated pneumonia, as defined by physician (crude OR 
Table 1. Baseline characteristics of patients treated with BBs pre-stroke vs. patients not treated with BB before stroke

\begin{tabular}{|c|c|c|}
\hline & $\begin{array}{l}\text { BB before stroke } \\
(\mathrm{n}=885)\end{array}$ & $\begin{array}{l}\text { No BB before stroke } \\
(\mathrm{n}=1,648)\end{array}$ \\
\hline Age, years, median (interquartile range) & $77(69-83)$ & $71(60-80)$ \\
\hline Gender, male, $\mathrm{n}(\%)$ & $476 / 885(5)$ & $965 / 1,648(6)$ \\
\hline \multicolumn{3}{|l|}{ History, n (\%) } \\
\hline Atrial fibrillation/flutter & $248 / 884(28)$ & $143 / 1,648(9)$ \\
\hline Stroke & $357 / 884(40)$ & $468 / 1,648(28)$ \\
\hline Hypercholesterolemia & $320 / 875(37)$ & $343 / 1,640(21)$ \\
\hline Hypertension & $695 / 881(79)$ & $700 / 1,648(42)$ \\
\hline Myocardial infarction & $207 / 884(23)$ & $123 / 1,648(7)$ \\
\hline Cardiac valve disease & $104 / 885(12)$ & $68 / 1,646(4)$ \\
\hline Peripheral vascular disease & 95/883 (11) & $93 / 1,642(6)$ \\
\hline Obstructive pulmonary disease & $79 / 885(9)$ & $129 / 1,644(8)$ \\
\hline Immunocompromise & $25 / 885(3)$ & $59 / 1,648(4)$ \\
\hline Current smoker, n (\%) & $157 / 871(18)$ & $462 / 1,634(28)$ \\
\hline \multicolumn{3}{|l|}{ Prior medication, $\mathrm{n}(\%)$} \\
\hline Anticoagulants & $177 / 885(20)$ & $105 / 1,647(6)$ \\
\hline Antiplatelet & $491 / 885(55)$ & $524 / 1,647(32)$ \\
\hline Statin & $480 / 884(54)$ & $464 / 1,647(28)$ \\
\hline ACE-inhibitor & $339 / 882(38)$ & $304 / 1,648(18)$ \\
\hline Proton pump inhibitor & $347 / 883(39)$ & $308 / 1,647(19)$ \\
\hline mRS score & $0(0-1)$ & $0(0-1)$ \\
\hline NIHSS & $5(3-10)$ & $5(3-9)$ \\
\hline Dysphagia, n (\%) & $237 / 832(28)$ & $384 / 1,534(25)$ \\
\hline Bladder catheter, n (\%) & $176 / 881(20)$ & $277 / 1,645(17)$ \\
\hline Thrombolysis, n (\%) & $284 / 885(32)$ & $550 / 1,648(33)$ \\
\hline Randomization to ceftriaxone, n (\%) & $428 / 885(48)$ & $838 / 1,648(51)$ \\
\hline Stroke type (hemorrhagic stroke vs. other), n (\%) & $76 / 885(9)$ & $192 / 1,648(12)$ \\
\hline
\end{tabular}

Table 2. Infection rate and outcome in patients using BB before stroke vs. patients not using BB before stroke

\begin{tabular}{|c|c|c|c|}
\hline & $\begin{array}{l}\text { BB before stroke } \\
(\mathrm{n}=885)\end{array}$ & $\begin{array}{l}\text { No BB before stroke } \\
(\mathrm{n}=1,648)\end{array}$ & OR $(95 \% \mathrm{CI})$ \\
\hline \multicolumn{4}{|l|}{ Physician diagnosis, $n(\%)$} \\
\hline Infection & $168 / 885(19)$ & $179 / 1,648(11)$ & $1.92(1.53-2.42)$ \\
\hline Pneumonia & $79 / 885(9)$ & $80 / 1,648(5)$ & $1.92(1.39-2.65)$ \\
\hline UTI & $79 / 885(9)$ & $93 / 1,848(5)$ & $1.64(1.20-2.24)$ \\
\hline Other infection & $23 / 885(3)$ & $27 / 1,648(2)$ & $1.60(0.91-2.81)$ \\
\hline \multicolumn{4}{|l|}{ Expert panel diagnosis, $n(\%)$} \\
\hline Infection & $62 / 885(7)$ & $67 / 1,648(4)$ & $1.78(1.25-2.54)$ \\
\hline Pneumonia & $30 / 885(3)$ & $27 / 1,648(2)$ & $2.11(1.24-3.57)$ \\
\hline UTI & $33 / 885(4)$ & $43 / 1,648(3)$ & $1.45(0.91-2.29)$ \\
\hline Other infection & $4 / 885(0.5)$ & $5 / 1,648(0.3)$ & $1.49(0.40-5.57)$ \\
\hline \multicolumn{4}{|l|}{ Outcome, $n(\%)$} \\
\hline Mortality at discharge & $85 / 884(9.6)$ & $60 / 1,647(3.6)$ & $1.85(1.28-2.70)$ \\
\hline Mortality at 3 months & $123 / 880(14.0)$ & $144 / 1,639(8.8)$ & $1.67(1.30-2.17)$ \\
\hline Unfavorable outcome at 3 months & $392 / 880(45)$ & $592 / 1,629(36)$ & $1.41(1.19-1.66)$ \\
\hline
\end{tabular}


Table 3. Risk factors for infection (physician diagnosis)

\begin{tabular}{|c|c|c|c|c|}
\hline Characteristic & Infection $(n=348)$ & $\begin{array}{l}\text { No infection } \\
(\mathrm{n}=2,190)\end{array}$ & $\begin{array}{l}\text { Multivariate analysis } \\
\text { aOR }(95 \% \mathrm{CI})\end{array}$ & $\mathrm{p}$ value \\
\hline Age, years, median (interquartile range) & $79.5(72-86)$ & $72(62-80)$ & $1.04(1.02-1.05)$ & $<0.01$ \\
\hline \multicolumn{5}{|l|}{ History, \% (n) } \\
\hline Atrial fibrillation/flutter & $23(81 / 348)$ & $14(310 / 2,190)$ & & \\
\hline Stroke & $34(119 / 348)$ & $32(708 / 2,190)$ & & \\
\hline Hypertension & $63(219 / 348)$ & $54(1,181 / 2,190)$ & & \\
\hline Myocardial infarction & $15(53 / 348)$ & $13(278 / 2,190)$ & & \\
\hline Cardiac valve disease & $7(24 / 348)$ & $7(149 / 2,190)$ & & \\
\hline Peripheral vascular disease & $11(37 / 348)$ & $7(153 / 2,190)$ & $1.76(1.09-2.84)$ & 0.02 \\
\hline Obstructive pulmonary disease & $11(38 / 348)$ & $8(170 / 2,190)$ & & \\
\hline Diabetes mellitus & $23(81 / 348)$ & $19(421 / 2,190)$ & & \\
\hline \multicolumn{5}{|l|}{ Prior medication, \% (n) } \\
\hline Anticoagulants & $15(52 / 348)$ & $11(231 / 2,190)$ & & \\
\hline Antiplatelet therapy & $44(153 / 348)$ & $39(865 / 2,190)$ & & \\
\hline Statins & $39(134 / 348)$ & $37(815 / 2,190)$ & & \\
\hline ACE-inhibitors & $29(100 / 348)$ & $25(544 / 2,190)$ & & \\
\hline BBs & $48(168 / 348)$ & $33(717 / 2,190)$ & $1.61(1.19-2.18)$ & 0.01 \\
\hline Proton pump inhibitors & $27(95 / 348)$ & $26(560 / 2,190)$ & & \\
\hline $\mathrm{mRS}$ & $0(0-2)$ & $0(0-1)$ & & \\
\hline NIHSS & $11(6-16)$ & $4(3-8)$ & $1.09(1.06-1.12)$ & $<0.01$ \\
\hline Dysphagia, \% (n) & $60(186 / 310)$ & $21(437 / 2,061)$ & $2.90(2.10-4.01)$ & $<0.01$ \\
\hline Bladder catheter, \% (n) & $52(181 / 347)$ & $12(272 / 2,184)$ & $3.95(2.91-5.36)$ & $<0.01$ \\
\hline Stroke type (bleeding vs. other), \% (n) & $17(60 / 348)$ & $10(209 / 2,190)$ & $1.79(1.14-2.82)$ & 0.01 \\
\hline
\end{tabular}

$1.92,95 \%$ CI $1.39-2.65 ; \mathrm{p}<0.001)$. Advanced age, ethnicity, history of atrial fibrillation/flutter, obstructive pulmonary disease, malignancy, current smoking status, diastolic and systolic blood pressure, score on NIHSS, disability prior to symptoms (mRS), dysphagia, coagulant therapy, and stroke type were also associated with stroke-associated pneumonia in univariate analysis. After correction for these factors in a multivariable analysis, the aOR for $\mathrm{BBs}$ and pneumonia was 1.56 (95\% CI 1.05-2.30; $\mathrm{p}=0.03$ ). Analyses using the expert panel definition for pneumonia showed a similar trend for BBs use and increased risk of stroke-associated pneumonia $(\mathrm{aOR} 1.76,95 \%$ CI $0.92-3.36$; $\mathrm{p}=$ 0.09).

The crude OR for BBs and diagnosis of UTI as defined by the physician was 1.64 (95\% CI 1.20-2.34; p < 0.01). Advanced age, male sex, atrial fibrillation/flutter, hypertension and hypercholesterolemia, current smoking sta-

Pre-Stroke Use of BBs Does Not Lower

Post-Stroke Infection Rate tus, stroke severity, presence of bladder catheter, treatment with thrombolysis, stroke type, and randomization were included in the multivariate analysis, showing an aOR for BBs and UTI of 1.25 (95\% CI 0.85-1.83; $\mathrm{p}=0.25$ ).

Baseline BB use was associated with an unfavorable outcome (table 3 ), but these associations did not remain significant after correction for other prognostic variables as mentioned in the methods section (aOR for mortality at 3 months $1.14,95 \%$ CI $0.84-1.53$; $\mathrm{p}=0.41$; aOR for unfavorable outcome at 3 months $1.10,95 \%$ CI $0.89-1.35$; $\mathrm{p}=0.39$ ).

\section{Discussion}

In our analysis, BBs were not protective for poststroke infection. In contrast to previous studies, we found that baseline use of BBs was associated with a 
higher risk for infection. The previous 4 studies on $\mathrm{BB}$ treatment and infection risk reported that BBs were either associated with decreased infection risk or there was no association $[4,5,8,9]$. All studies had a retrospective study design and were heterogeneous with respect to stroke type (ischemic or hemorrhagic) and definitions of BB use (prior to stroke or after stroke admission) and infections. In the previous 4 studies, definition of infection was not described in 2 studies. It was based on adverse event recording in one study, and in the other study modified CDC criteria were used. Our study had a prospective study design and predefined definition of infection, according to international consensus [10]. Data on BB treatment was prospectively collected and our large sample gave us the statistical power to perform multivariable analysis.

Baseline use of BBs was associated with a higher risk for infection. However, patients on BBs were older, more often had comorbidities, and used more medication than patients not on BBs. It has been well-recognized that patients with advanced age are more vulnerable for infections than previously healthy patients. Also, some comorbidities and medications are associated with infection $[11,12]$. By performing multivariate analysis, we tried to correct this higher baseline infection risk in BB-treated patients, but it is possible that confounding by indication might have influenced the results. Also, mortality rate was higher in patients treated with BBs. This introduces a competing risk bias: deceased patients are not at risk of infection.

The etiology of stroke-associated infection is multifactorial. Infection occurs more often in patients with more severe stroke and advanced age; dysphagic patients are at a high risk for pneumonia, and patients with indwelling catheter for UTI. Also, post-stroke immune suppression, which could be mediated by hypothalamo-pituitaryadrenal axis and sympathetic nervous system activation, increases the infection risk $[2,3,12]$. Stroke-associated respiratory syndrome includes pneumonia, but also respiratory tract infections without chest-X-ray abnormalities and even a subset of these syndromes could be inflammatory rather than infective [13]. In a recent consensus of the 'Pneumonia in Stroke Consensus Group', it was agreed that the spectrum of lower-respiratory-tract-infections in the first 7 days after acute stroke are named as stroke-associated-pneumonia. In this study, these criteria for diagnosis were used. From previous experimental and clinical studies, it is thought that adrenergic effects on peripheral blood immune cells could enhance immune suppression and increase the infection risk, and BBs have the potential to diminish these effects [14]. The results of this study do not support such an effect for pre-stroke use of BBs. Yet, effects of BBs have been shown to be dose dependent, and dosage dependent effects could have been missed since the dosage of BB therapy was not controlled in this study [15]. Also, effects might differ between BBs already used prior to stroke, as compared to BBs started directly after stroke [5]. Only a randomized clinical trial could investigate the true potential of $\mathrm{BB}$ treatment for reducing stroke-associated infections, but the results of this study are not encouraging.

This study has several limitations. First, only prestroke $\mathrm{BB}$ use was investigated. In previous studies, stronger associations were found for on-stroke treatment with BB. Second, use of BBs was strictly defined, but the class of drug, dose, and compliance of $\mathrm{BB}$ use at baseline was not. This treatment was recorded by the physician in a prospective manner, but the treatment itself, including dosage, was not recorded. In the Netherlands, it is a standard practice to continue antihypertensive medication used prior to stroke during hospital stay after stroke, and in the PASS no standard protocol was used for discontinuation of antihypertensive treatment during admission. Any discontinuation of treatment after randomization could theoretically have led to an underestimation of the effect of BBs, and dosage dependent effects could have been missed. Also, we did not distinguish between selective and non-selective BBs. These different classes could have differing working mechanisms; however, for both groups associations with immune response and infection have been described [4]. Third, this study is a cohort study, which contains the risk of selection bias. The population of the PASS had relatively mild stroke and a low rate of infection. This could theoretically have diminished the potential of the effect of BB therapy, but, since the infection rate was unchanged or even higher in $\mathrm{BB}$ treated patients, such an effect is unlikely. Finally, we were able to perform multivariable analysis because of the large study population, however because diagnosis of pneumonia by expert panel was made in a limited number of patients, this multivariable analysis contained more variables than statistically appropriate and should therefore be interpreted with caution.

\section{Conclusion}

Patients treated with BBs prior to stroke have a higher rate of infection and pneumonia, but not of UTI. 


\section{Sources of Funding}

The PASS study was funded by the Academic Medical Centre, by the Netherlands Organization for Health Research and Development (ZonMW; 171002302), and the Netherlands Heart Foundation (Hartstichting; 2009B095). Principal investigators of the PASS are Dr. P.J. Nederkoorn and Professor D. van de Beek. D.B. is supported by grants from the European Research Council (ERC Starting Grant (Proposal/Contract number 281156)), Netherlands Organization for Health Research and Development (ZonMw; NWO-Vidi grant 2010 (Proposal/Contract number 016.116.358)).

\section{Disclosure Statement}

The authors declare that they have no conflicts of interest.

\section{Authors Contributions}

W.F.W. wrote this manuscript together with D.B. and J.-D.V., M.C.B., Y.B.W.E.M.R. and P.J.N. commented on the paper. All authors agreed to the final version. All authors were involved in the design of the PASS and collection of data.

\section{References}

1 Popović N, Stefanović-Budimkić M, Mitrović $N$, Urošević A, Milošević B, Pelemiš $M$, Jevtović D, Beslać-Bumbaširević L, Jovanović $D$ : The frequency of poststroke infections and their impact on early stroke outcome. J Stroke Cerebrovasc Dis 2013;22:424-429.

-2 Chamorro Á, Meisel A, Planas AM, Urra X, van de Beek D, Veltkamp R: The immunology of acute stroke. Nat Rev Neurol 2012;8:401410.

3 Wong $\mathrm{CH}$, Jenne $\mathrm{CN}$, Lee WY, Léger C, Kubes P: Functional innervation of hepatic iNKT cells is immunosuppressive following stroke. Science 2011;334:101-105.

4 Maier IL, Karch A, Mikolajczyk R, Bähr M, Liman J: Effect of beta-blocker therapy on the risk of infections and death after acute stroke - a historical cohort study. PLoS One 2015;10:e0116836.

5 Sykora M, Siarnik P, Diedler J; VISTA Acute Collaborators: $\beta$-Blockers, pneumonia, and outcome after ischemic stroke: evidence from virtual international stroke trials archive. Stroke 2015;46:1269-1274.

-6 Westendorp WF, Vermeij JD, Zock E, Hooijenga IJ, Kruyt ND, Bosboom HJ, Kwa VI,
Weisfelt M, Remmers MJ, ten Houten R, Schreuder AH, Vermeer SE, van Dijk EJ, Dippel DW, Dijkgraaf MG, Spanjaard L, Vermeulen M, van der Poll T, Prins JM, Vermeij FH, Roos YB, Kleyweg RP, Kerkhoff H, Brouwer MC, Zwinderman AH, van de Beek D, Nederkoorn PJ; PASS Investigators: The preventive antibiotics in stroke study (PASS): a pragmatic randomised open-label masked endpoint clinical trial. Lancet 2015;385: 1519-1526.

7 Horan TC, Andrus M, Dudeck MA: CDC/ NHSN surveillance definition of health careassociated infection and criteria for specific types of infections in the acute care setting. Am J Infect Control 2008;36:309-332.

-8 Dziedzic T, Slowik A, Pera J, Szczudlik A: Beta-blockers reduce the risk of early death in ischemic stroke. J Neurol Sci 2007;252:53-56.

-9 Kalita J, Misra UK, Kumar B: Is $\beta$-blocker (atenolol) a preferred antihypertensive in acute intracerebral hemorrhage? Neurol Sci 2013; 34:1099-1104.

10 Smith CJ, Kishore AK, Vail A, Chamorro A, Garau J, Hopkins SJ, Di Napoli M, Kalra L, Langhorne P, Montaner J, Roffe C, Rudd AG,
Tyrrell PJ, van de Beek D, Woodhead M, Meisel A: Diagnosis of stroke-associated pneumonia: recommendations from the pneumonia in stroke consensus group. Stroke 2015;46:2335-2340.

11 Kwan J, Hand P: Infection after acute stroke is associated with poor short-term outcome. Acta Neurol Scand 2007;115:331-338.

12 Emsley HC, Hopkins SJ: Post-stroke immunodepression and infection: an emerging concept. Infect Disord Drug Targets 2010;10: 91-97.

13 Marik PE: Aspiration pneumonitis and aspiration pneumonia. N Engl J Med 2001;344: 665-671.

14 Griffin GD: Stroke, mTBI, infection, antibiotics and beta blockade: connecting the dots. Med Hypotheses 2015;85:224-229.

15 Prass K, Meisel C, Höflich C, Braun J, Halle E, Wolf T, Ruscher K, Victorov IV, Priller J, Dirnagl U, Volk HD, Meisel A: Stroke-induced immunodeficiency promotes spontaneous bacterial infections and is mediated by sympathetic activation reversal by poststroke T helper cell type 1-like immunostimulation. J Exp Med 2003;198:725-736. 